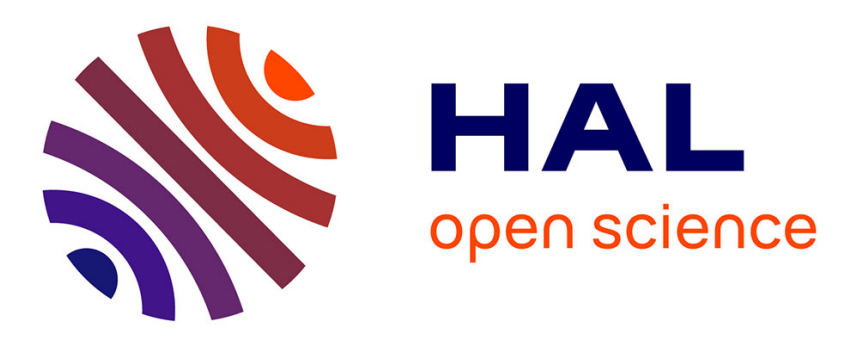

\title{
Multiple equilibria in two-sector monetary economies: an interplay between preferences and the timing for money
}

Stefano Bosi, Kazuo Nishimura, Alain Venditti

\section{- To cite this version:}

Stefano Bosi, Kazuo Nishimura, Alain Venditti. Multiple equilibria in two-sector monetary economies: an interplay between preferences and the timing for money. 2009. halshs-00432258

\author{
HAL Id: halshs-00432258 \\ https://shs.hal.science/halshs-00432258 \\ Preprint submitted on 15 Nov 2009
}

HAL is a multi-disciplinary open access archive for the deposit and dissemination of scientific research documents, whether they are published or not. The documents may come from teaching and research institutions in France or abroad, or from public or private research centers.
L'archive ouverte pluridisciplinaire HAL, est destinée au dépôt et à la diffusion de documents scientifiques de niveau recherche, publiés ou non, émanant des établissements d'enseignement et de recherche français ou étrangers, des laboratoires publics ou privés. 


\title{
GREQAM
}

Document de Travail

Groupement de Recherche en Economie Quantitative d'Aix-Marseille - UMR-CNRS 6579 Ecole des Hautes Etudes en Sciences Sociales Universités d'Aix-Marseille II et III

MULTIPLE EQUILIBRIA IN TWO-SECTOR MONETARY ECONOMIES : AN INTERPLAY BETWEEN PREFERENCES AND THE TIMING FOR MONEY

\author{
Stefano BOSI \\ Kazuo NISHIMURA \\ Alain VENDITTI
}

September 2009 


\title{
Multiple equilibria in two-sector monetary economies: an interplay between preferences and the timing for money
}

\author{
Stefano BOSI \\ EQUIPPE, University of Lille \\ E-mail: stefano.bosi@orange.fr \\ Kazuo NISHIMURA \\ Institute of Economic Research, Kyoto University \\ E-mail: nishimura@kier.kyoto-u.ac.jp \\ and \\ Alain VENDITTI \\ CNRS - GREQAM and EDHEC \\ E-mail: Alain.Venditti@univmed.fr
}

First version: November 2007; Revised: January 2009

\begin{abstract}
In this paper, we study the occurrence of local indeterminacy in two-sector monetary economies. In order to capture the credit market imperfections and the liquidity services of money, we consider a general MIUF model with two alternative timings in monetary payments: the Cash-In-Advance timing, in which the cash available to buy goods is money in the consumers' hands after they leave the bond market but before they enter the goods market, and the Cash-After-the-Market timing, in which agents hold money for transactions after leaving the goods market. We consider three standard specifications of preferences: the additively separable formulation, the Greenwood-Hercovitz-Huffman (GHH) [18] formulation and the King-Plosser-Rebelo (KPR) [21] formulation. First, we show that for all the three types of preferences, local indeterminacy easily arises under the CIA timing with a low enough interest rate elasticity of money demand. Second, we show that with the CAM timing, determinacy always holds under separable preferences, but local indeterminacy can arise in the case of GHH and KPR preferences. We thus prove that compared to aggregate models, two-sector models provide new rooms for local indeterminacy when non-separable standard preferences are considered.
\end{abstract}

Keywords: Money-in-the-utility-function, Indeterminacy, Sunspot equilibria.

Journal of Economic Literature Classification Numbers: C61, E32. 


\section{Introduction}

A wide-spread perception among economists is that macroeconomic businesscycle fluctuations are not driven only by shocks on technologies or preferences, but also by changes in expectations about the fundamentals. The 2008 financial crisis is a good illustration to show how coordination failures in the monetary market and self-fulfilling prophecies can have dramatic effects either on the stock or the goods market, and account for the observed macroeconomic fluctuations to a large extent.

A major strand of the literature focussing on fluctuations derived from agents' beliefs is based on the concept of sunspot equilibria that dates back to the early works of Shell [26], Azariadis [1] and Cass and Shell [12]. As shown by Woodford [30], the existence of sunspot equilibria is closely related to the equilibrium indeterminacy under perfect foresight, i.e. the existence of a continuum of equilibrium paths converging toward one steady state from the same initial value of the state variable.

It is well-known that equilibrium indeterminacy can arise in monetary models in which the central bank follows an exogenous money growth rule. ${ }^{1}$ Transactions need money and a standard approach consists in assuming that agents face borrowing constraints, demanding money and bearing the opportunity cost of a nominal interest rate. ${ }^{2}$ In order to capture the transactions motive for money holding, the Money-In-the-Utility-Function (MIUF) approach is widely used in the literature as it generalizes either the transactions cost or the shopping time models as shown by Feenstra [16]. ${ }^{3}$

However, the timing of transactions matters depending on the way real balances enter the utility function. On the one hand, with the Cash-InAdvance (CIA) timing, the cash available to buy goods is money in the consumers' hands after they leave the bond market but before they enter the goods market. Under this timing and a Leontief utility function, the MIUF model reduces to the Clower [13] constraint. ${ }^{4}$ Within aggregate models, local indeterminacy easily arises under a CIA timing and requires a sufficiently weak elasticity of intertemporal substitution, together with dominant income effects, i.e. a low enough interest elasticity of money demand. ${ }^{5}$

\footnotetext{
${ }^{1}$ See Michener and Ravikumar [24] for a survey.

${ }^{2}$ See for instance Barinci and Chéron [2], Woodford [31].

${ }^{3}$ See Farmer [15], Matsuyama [23].

${ }^{4}$ In the spirit of Clower, the reader is also referred to Grandmont and Younès [17] for a partial constraint, Stockman [28] for capital accumulation, Lucas and Stokey [22] for a cash-credit approach.

${ }^{5}$ See, Carlstrom and Fuerst [11], Cooley and Hansen [14].
} 
On the other hand, the traditional Sidrauski [27] view implies that end-ofperiod real balances enter the utility function. ${ }^{6}$ As agents hold money for transactions after leaving the goods market, this timing is called Cash-Afterthe-Market (CAM), and whithin an aggregate economy with production, it implies that indeterminacy never arises. ${ }^{7}$ The aim of our paper is to explore the robustness of those conclusions within a monetary two-sector economy.

The main motivation to consider a two-sector model is that local indeterminacy has been shown to occur much more easily in such a framework than within aggregate models. The shifting of resources between the productions of the two goods provides indeed new mechanisms through the implied variations of prices. This is true at the same time in real models with productive externalities, ${ }^{8}$ and in monetary models with fractional CIA constraint. ${ }^{9}$ In this paper, we investigate whether two-sector models also provide new rooms for local indeterminacy when a general MIUF formulation is considered.

While the production side is based on the consideration of general technologies, the side of preferences is defined on the ground of three different specifications of the utility function which are commonly used in the business cycle and growth literature: i) an additively separable formulation, ii) a King, Plosser and Rebelo [21] (KPR) formulation which is compatible with balanced growth, and finally iii) a Greenwood, Hercovitz and Huffman [18] (GHH) formulation for which the marginal rate of substitution between consumption and real money balances depends on the latter only. The CIA and CAM timings are systematically compared throughout the paper. Considering the CIA timing, we first generalize the conclusions obtained by Bosi, Magris and Venditti [9] in a two-sector economy with CIA constraint : local indeterminacy requires a low enough interest rate elasticity of money demand. ${ }^{10}$ Second, and contrary to the aggregate formulation, we highlight the possible existence of local indeterminacy in two-sector monetary economies with a CAM timing depending on the utility function. While we show that the determinacy result is robust under additively separable preferences, we prove that this is no longer the case with non-separable preferences by providing simple sufficient conditions for local indeterminacy

\footnotetext{
${ }^{6}$ See also Brock [10].

${ }^{7}$ See Carlstrom and Fuerst [11] in which this timing is called Cash-When-I'm-Done.

${ }^{8}$ See for instance Benhabib and Nishimura [6] in which it is shown that local indeterminacy arises under constant social returns to scale if there is a capital intensity reversal between the private and the social levels.

${ }^{9}$ See for instance Bosi, Magris and Venditti [8] in which it is shown that local indeterminacy occurs without any restriction on the utility function is the share of liquidity constraint is low enough, i.e. if the stationary velocity of money is large enough.

${ }^{10}$ The same restriction is necessary in aggregate models (see Carlstrom and Fuerst [11]).
} 
in the case of GHH [18] and KPR [21] utility functions. Explicit examples with CES technologies illustrate these theoretical findings. Summing up, the novelty of the paper not only rests on a more general (bi-sectoral) MIUF approach to the CIA timing where indeterminacy more likely arises, but also on the occurrence of indeterminacy under a CAM timing (never shown in literature) with heterogeneous technologies and non-separable preferences.

The paper is organized as follows. The next section sets up the basic model, defines the intertemporal equilibrium and proves the existence of a steady state. In Section 3, we present our main results by focussing successively on additively separable, GHH and KPR preferences. Section 5 illustrates our conclusions through a simple example. Section 6 contains concluding comments and all the proofs are in a final Appendix.

\section{The model}

\subsection{Technology}

The basic model is a two-sector optimal growth model. We assume that there are two commodities with one consumption good $y_{0}$ and one capital good $y$. The labor supply is assumed to be inelastic. Total labor is normalised to 1 and each good is produced with a constant returns to scale technology:

$$
y_{0}=f^{0}\left(k_{0}, l_{0}\right), \quad y=f^{1}\left(k_{1}, l_{1}\right)
$$

with $k_{0}+k_{1} \leq k, k$ being the total stock of capital, and $l_{0}+l_{1} \leq 1$.

Assumption 1. Each production function $f^{i}: \mathbb{R}_{+}^{2} \rightarrow \mathbb{R}_{+}, i=0,1$, is $C^{2}$, increasing in each argument, concave, homogeneous of degree one and such that for any $x>0, f_{1}^{i}(0, x)=f_{2}^{i}(x, 0)=+\infty, f_{1}^{i}(+\infty, x)=f_{2}^{i}(x,+\infty)=0$.

By definition we have $y \leq f^{1}(k, 1)$. The monotonicity properties and the Inada conditions in Assumption 1 imply that there exists $\bar{k}>0$ solution of $k-f^{1}(k, 1)=0$ such that $f^{1}(k, 1)>k$ when $k<\bar{k}$, and $f^{1}(k, 1)<k$ when $k>\bar{k}$. It follows that it is not possible to maintain stocks beyond $\bar{k}$. The set of admissible pairs $(k, y)$ is thus defined as follows

$$
\mathcal{D}=\left\{(k, y) \in \mathbb{R}_{+}^{2} \mid 0 \leq k \leq \bar{k}, 0 \leq y \leq f^{1}(k, 1)\right\}
$$

There are two representative firms, one for each sector. For any given $(k, y)$, profit maximization is equivalent to solving the following problem of optimal allocation of productive factors between the two sectors: 


$$
\begin{aligned}
T(k, y)=\max _{k_{0}, k_{1}, l_{0}, l_{1}} & f^{0}\left(k_{0}, l_{0}\right) \\
\text { s.t. } & y \leq f^{1}\left(k_{1}, l_{1}\right) \\
& k_{0}+k_{1} \leq k \\
& l_{0}+l_{1} \leq 1 \\
& k_{0}, k_{1}, l_{0}, l_{1} \geq 0
\end{aligned}
$$

The social production function $T(k, y)$ describes the frontier of the production possibility set associated with interior temporary equilibria such that $(k, y) \in \mathcal{D}$, and gives the maximal output of the consumption good. Under Assumption 1, for any $(k, y) \in \mathcal{D}, T(k, y)$ can be shown to be concave and twice continuously differentiable. ${ }^{11}$ Denoting $w$ the wage rate, $r$ the gross rental rate of capital and $p$ the price of investment good, all in terms of the price of the consumption good, we formulate the aggregate profit maximization problem as follows

$$
\max _{(k, y) \in \mathcal{D}} T(k, y, \ell)+p y-r k-w \ell
$$

and we derive that for any $(k, y) \in \operatorname{int} \mathcal{D}$, with $\operatorname{int} \mathcal{D}$ denoting the interior of the set $\mathcal{D}$, the first order derivatives of the social production function give

$$
T_{1}(k, y)=r, \quad T_{2}(k, y)=-p
$$

Buiding on the constant returns to scale, it follows that the wage rate satisfies $T(k, y)+p y=r k+w$.

\section{$2.2 \quad$ Preferences}

The economy is populated by a large number of identical infinitely-lived agents. We will assume without loss of generality that the total population is constant. In each period $t \geq 0$, each consumer receives income from capital, labor, money and lump-sum transfers from the government. His expenditures concern the consumption good, the investment good and money for the next period $t+1$. Let us then denote by $M_{t}$ the stock of money at time $t$. In each period $t \geq 0$, the representative agent is subject to the budget constraint

$$
\hat{q}_{t} c_{t}+\hat{p}_{t} y_{t}+M_{t+1}=\hat{r}_{t} k_{t}+\hat{w}_{t}+M_{t}+\hat{\tau}_{t}
$$

where $\hat{q}_{t}$ is the price of the consumption good, $\hat{p}_{t}$ is the price of the investment good, $\hat{r}_{t}$ is the rental rate of capital, $\hat{w}_{t}$ is the wage rate and $\hat{\tau}$ is the

\footnotetext{
${ }^{11}$ See Benhabib and Nishimura [4]
} 
nominal lump-sum transfers issued by the government. Choosing the consumption good as the numéraire, i.e. dividing equation (5) by $\hat{q}_{t}$, gives the following formulation for the budget constraint

$$
c_{t}+p_{t} y_{t}+q_{t} M_{t+1}=r_{t} k_{t}+w_{t}+q_{t} M_{t}+\tau_{t}
$$

with $p_{t}=\hat{p}_{t} / \hat{q}_{t}, q_{t}=1 / \hat{q}_{t}, r_{t}=\hat{r}_{t} / \hat{q}_{t}, w_{t}=\hat{w}_{t} / \hat{q}_{t}$ and $\tau_{t}=\hat{\tau}_{t} / \hat{q}_{t}$. Money in real terms is thus given by $q_{t} M_{t}$.

The per-period utility function $u\left(c_{t}, q_{t} \mathcal{M}_{t}\right)$ depends on consumption $c_{t}$ and real money balances denoted now $q_{t} \mathcal{M}_{t}$. In the literature, there are indeed two typical choices for the formulation of the cash that facilitates trading at time $t$. The first one, used for instance by Woodford [31], corresponds to the cash the household has in advance of goods-market trading, namely

$$
\mathcal{M}_{t}=M_{t}
$$

and is called Cash-In-Advance (CIA) timing. The second formulation, used for instance by Matsuyama [23], corresponds to the cash left to the household after leaving the goods market, namely

$$
\mathcal{M}_{t}=M_{t+1}
$$

and is called Cash-After-the-Market (CAM) timing.

Considering that $\mathcal{M}_{t}=M_{t}$ or $M_{t+1}$, the per-period utility function satisfies the following basic restrictions:

Assumption 2. $u(c, q \mathcal{M})$ is $C^{2}$, increasing in each argument, concave and satisfies the boundary conditions $\lim _{q \mathcal{M} \rightarrow 0} u_{2}(x, q \mathcal{M}) / u_{1}(x, q \mathcal{M})=+\infty$, $\lim _{q \mathcal{M} \rightarrow+\infty} u_{2}(x, q \mathcal{M}) / u_{1}(x, q \mathcal{M})=0$ for any $x>0$.

We also introduce a standard normality assumption between consumption $c$ and real money balances $q \mathcal{M}$

Assumption 3. Consumption $c$ and real money balances $q \mathcal{M}$ are normal goods.

The consumption of the representative agent at time $t$ is given by $c_{t}=$ $T\left(k_{t}, y_{t}\right)$, and the capital accumulation equation is such that

$$
k_{t+1}=y_{t}+(1-\delta) k_{t}
$$

with $\delta \in[0,1]$ the rate of depreciation of capital. The intertemporal maximisation program of the representative agent is then as follows 


$$
\begin{array}{cl}
\max _{\left\{c_{t}, k_{t+1}, M_{t+1}\right\}_{t=0}^{+\infty}} & \sum_{t=0}^{+\infty} \beta^{t} u\left(c_{t}, q_{t} \mathcal{M}_{t}\right) \\
\text { s.t. } & c_{t}+p_{t}\left[k_{t+1}-(1-\delta) k_{t}\right]+q_{t} M_{t+1}=r_{t} k_{t}+w_{t}+q_{t} M_{t}+\tau_{t} \\
& k_{0}, M_{0} \text { given }
\end{array}
$$

where $\beta \in(0,1]$ denotes the discount factor, and $\mathcal{M}_{t}=M_{t}$ or $M_{t+1}$.

\subsection{Intertemporal equilibrium}

The generalised Lagrangian is

$$
\begin{aligned}
\mathcal{L} & =\sum_{t=0}^{+\infty} \beta^{t} u\left(c_{t}, q_{t} \mathcal{M}_{t}\right) \\
& +\sum_{t=0}^{+\infty} \beta^{t} \lambda_{t}\left[r_{t} k_{t}+w_{t}+q_{t} M_{t}+\tau_{t}-c_{t}-p_{t}\left[k_{t+1}-(1-\delta) k_{t}\right]-q_{t} M_{t+1}\right]
\end{aligned}
$$

with $\lambda$ the non-negative Lagrange multiplier associated with constraint (6). With both timings, the first order conditions with respect to $k_{t+1}$ is identical and given by

$$
\lambda_{t} p_{t}=\beta \lambda_{t+1}\left[r_{t+1}+(1-\delta) p_{t+1}\right]
$$

where $r_{t}$ and $p_{t}$ are defined by (4). However, the first order conditions with respect to $c_{t}$ and $\mathcal{M}_{t}$ depend on the timing and are given by

$$
\begin{aligned}
\lambda_{t} & =u_{1}\left(c_{t}, q_{t} M_{t}\right) \\
\lambda_{t} q_{t} & =\beta q_{t+1}\left[\lambda_{t+1}+u_{2}\left(c_{t+1}, q_{t+1} M_{t+1}\right)\right]
\end{aligned}
$$

with the CIA timing, or

$$
\begin{aligned}
\lambda_{t} & =u_{1}\left(c_{t}, q_{t} M_{t+1}\right) \\
\beta \lambda_{t+1} q_{t+1} & =q_{t}\left[\lambda_{t}-u_{2}\left(c_{t}, q_{t} M_{t+1}\right)\right]
\end{aligned}
$$

with the CAM timing.

By manipulating the first order conditions (10)-(14), we obtain the Euler equation and the arbitrage condition between consumption and real balances

$$
\begin{aligned}
& u_{1}\left(c_{t}, q_{t} M_{t}\right) p_{t}=\beta u_{1}\left(c_{t+1}, q_{t+1} M_{t+1}\right)\left[r_{t+1}+(1-\delta) p_{t+1}\right] \\
& u_{1}\left(c_{t}, q_{t} M_{t}\right) q_{t}=\beta q_{t+1}\left[u_{1}\left(c_{t+1}, q_{t+1} M_{t+1}\right)+u_{2}\left(c_{t+1}, q_{t+1} M_{t+1}\right)\right]
\end{aligned}
$$

with the CIA timing, or

$$
\begin{aligned}
u_{1}\left(c_{t}, q_{t} M_{t+1}\right) p_{t} & =\beta u_{1}\left(c_{t+1}, q_{t+1} M_{t+2}\right)\left[r_{t+1}+(1-\delta) p_{t+1}\right] \\
\beta u_{1}\left(c_{t+1}, q_{t+1} M_{t+2}\right) q_{t+1} & =q_{t}\left[u_{1}\left(c_{t}, q_{t} M_{t+1}\right)-u_{2}\left(c_{t}, q_{t} M_{t+1}\right)\right]
\end{aligned}
$$

with the CAM timing.

In order to complete the definition of the intertemporal equilibrium, we need to specify the supply of money. We assume that the government follows 
a simple monetary rule: in each period it issues lump-sum transfers of money balances at the constant rate $\mu-1>0$, so that in period $t$ the supply of money $M_{t}^{s}$ satisfies $M_{t}^{s}=\mu^{t} M_{0}^{s}$, with $M_{0}^{s}=M_{0}$ the initial amount of money balances. Thus nominal transfers are given by $\tau_{t}=(\mu-1) q_{t} M_{t}^{s}$. Using the notations $m_{t}=q_{t} M_{t}$ and $z_{t}=q_{t} M_{t+1}$, we finally get from (15)-(16) and from the equilibrium on the monetary market $M_{t}^{s}=M_{t}$ :

$$
\begin{aligned}
u_{1}\left(c_{t}, m_{t}\right) p_{t} & =\beta u_{1}\left(c_{t+1}, m_{t+1}\right)\left[r_{t+1}+(1-\delta) p_{t+1}\right] \\
u_{1}\left(c_{t}, m_{t}\right) m_{t} & =\frac{\beta}{\mu}\left[u_{1}\left(c_{t+1}, m_{t+1}\right)+u_{2}\left(c_{t+1}, m_{t+1}\right)\right] m_{t+1}
\end{aligned}
$$

with the CIA timing, or

$$
\begin{aligned}
u_{1}\left(c_{t}, z_{t}\right) p_{t} & =\beta u_{1}\left(c_{t+1}, z_{t+1}\right)\left[r_{t+1}+(1-\delta) p_{t+1}\right] \\
\frac{\beta}{\mu} u_{1}\left(c_{t+1}, z_{t+1}\right) z_{t+1} & =z_{t}\left[u_{1}\left(c_{t}, z_{t}\right)-u_{2}\left(c_{t}, z_{t}\right)\right]
\end{aligned}
$$

with the CAM timing.

Taking into account $c_{t}=T\left(k_{t}, y_{t}\right), r_{t}=T_{1}\left(k_{t}, y_{t}\right), p_{t}=-T_{2}\left(k_{t}, y_{t}\right)$ and $y_{t}=k_{t+1}-(1-\delta) k_{t}$, we conclude that (17) and (18) are two systems of implicit difference equations of order 2 in the capital stock $k$ and order 1 in real money balances $m$ or $z$.

Any optimal plan for the representative household that satisfies the Euler equations (17) or (18) and the corresponding following transversality condition

$$
\begin{aligned}
\lim _{t \rightarrow+\infty} \beta^{t} u_{1}\left(c_{t}, x_{t}\right) p_{t} k_{t+1} & =0 \\
\lim _{t \rightarrow+\infty} \beta^{t} u_{1}\left(c_{t}, x_{t}\right) x_{t} & =0
\end{aligned}
$$

with $x_{t}=m_{t}$ or $z_{t}$, is called an equilibrium path.

\subsection{Steady state}

A steady state is defined as $k_{t}=k^{*}, y_{t}=y^{*}=\delta k^{*}, c_{t}=c^{*}, p_{t}=p^{*}=$ $-T_{2}\left(k^{*}, \delta k^{*}\right), r_{t}=r^{*}=T_{1}\left(k^{*}, \delta k^{*}\right)$ and $m_{t}=m^{*}$ or $z_{t}=z^{*}$ for all $t$. Along a stationary solution, we have indeed a decreasing path for the price of money balances $q_{t+1} / q_{t}=1 / \mu$ which is associated with the expansion of money supply $M_{t+1} / M_{t}=\mu$ so that at the steady state real money balances $m_{t}$ or $z_{t}$ remain constant. From the dynamical system (17) and the fact that $c=T(k, \delta k)$, the stationary levels of capital $k^{*}$ and real money balances $m^{*}$ or $z^{*}$ are obtained as solutions of

$$
\frac{r}{p}=-\frac{T_{1}(k, \delta k)}{T_{2}(k, \delta k)}=\beta^{-1}-(1-\delta)
$$


and

with the CIA timing, or

$$
\frac{u_{2}(T(k, \delta k), m)}{u_{1}(T(k, \delta k), m)}=\frac{\mu}{\beta}-1
$$

$$
\frac{u_{2}(T(k, \delta k), z)}{u_{1}(T(k, \delta k), z)}=1-\frac{\beta}{\mu}
$$

with the CAM timing.

As in the standard one-sector Ramsey model, we get the following result:

Proposition 1 . Under Assumptions 1-2, there exists a unique steady state $\left(k^{*}, m^{*}\right)$ or $\left(k^{*}, z^{*}\right)$ solution of equations (21)-(22) or (21)-(23).

The steady states $\left(k^{*}, m^{*}\right)$ and $\left(k^{*}, z^{*}\right)$ are called the Modified Golden Rule.

\section{Main results}

In order to study the local determinacy properties of the steady state, we need to compute the characteristic roots associated with the linearized Euler equations around the Modified Golden Rule. At any time $t$ we have one predeterminate variable, the current capital stock $k_{t}$, and two forward variables, the capital stock of the following period $k_{t+1}$ and the current real money balances $m_{t}$ or $z_{t}$. Local indeterminacy will be obtained if at least two characteristic roots are inside the unit circle.

Note from equations (21)-(22) that the steady-state $\left(k^{*}, x^{*}\right)$, with $x^{*}=$ $m^{*}$ or $z^{*}$, is defined as follows: the capital stock $k^{*}$ is only determined by the technological characteristics, the discount factor $\beta$ and the rate of depreciation of capital $\delta$. The properties of preferences only influence the stationary value of real money balances $x^{*}=m^{*}$ or $z^{*}$. Then for some given technologies and a given pair $(\beta, \delta)$, the value of $k^{*}$ is fixed and it is easy to consider variations of preferences in order to study the occurrence of bifurcations. This will be our strategy of proof in the sequel of the paper.

We are looking for the simplest sufficient conditions for the occurrence of local indeterminacy. In order to derive economically interpretable results, we introduce, following Benhabib and Nishimura [5], the relative capital intensity difference across sectors such that

$$
b=\frac{l_{1}}{y}\left(\frac{k_{1}}{l_{1}}-\frac{k_{0}}{l_{0}}\right)
$$

The sign of $b$ is positive if and only if the investment good is capital intensive. Note also that the demands for input $k_{0}, k_{1}, l_{0}, l_{1}$ when evaluated at the steady state are functions of the discount factor $\beta$ and the rate of depreciation of capital $\delta$. It follows that the capital intensity difference satisfies 
$b=b(\beta, \delta)$. We also define the elasticities of substitution of consumption and real money balances evaluated at the steady state ${ }^{12}$

$$
\begin{aligned}
\epsilon_{c c} & =-\xi_{c c}^{-1}=-\left(u_{11}^{*} c^{*} / u_{1}^{*}\right)^{-1}>0, \quad \epsilon_{x c}=-\xi_{x c}^{-1}=-\left(u_{21}^{*} c^{*} / u_{2}^{*}\right)^{-1} \\
\epsilon_{x x} & =-\xi_{x x}^{-1}=-\left(u_{22}^{*} x^{*} / u_{2}^{*}\right)^{-1}>0, \quad \epsilon_{c x}=-\xi_{c x}^{-1}=-\left(u_{12}^{*} x^{*} / u_{1}^{*}\right)^{-1}
\end{aligned}
$$

with $x=m$ or $z, u_{i}^{*}=u_{i}^{m}$ or $u_{i}^{z}, u_{i j}^{*}=u_{i j}^{m}$ or $u_{i j}^{z}, i, j=1,2$, and the following elasticities of the consumption good's output and the rental rate with respect to the capital stock, all evaluated at the steady state

$$
\varepsilon_{c k}=T_{1}^{*} k^{*} / T^{*}>0, \quad \varepsilon_{r k}=T_{11}^{*} k^{*} / T_{1}^{*}<0
$$

We will explore the existence of local indeterminacy with $C I A$ and $C A M$ timings considering successively different specifications for the utility function which are widely used in the Real Business Cycle literature. We will then show that the occurrence of business cycles based on self-fulfilling prophecies depends on an interplay between preferences and the timing for money.

\subsection{Additively separable preferences}

Consider the standard case of additively separable preferences.

Assumption 4. $\xi_{c m}=\xi_{m c}=\xi_{c z}=\xi_{z c}=0$

Let us first assume the CIA timing. We introduce the following critical value for the elasticity of intertemporal substitution in consumption:

$$
\bar{\epsilon}_{c c}=\frac{\varepsilon_{c k} 2 \beta(1+\beta) \theta^{2}}{\varepsilon_{r k}[1+(2-\delta) b][\beta+b[1+(1-\delta) \beta]]}
$$

with $\theta=[1-\beta(1-\delta)]^{-1}$.

Theorem 1. With the CIA timing, let Assumptions 1-4 hold. Then the modified golden rule $\left(k^{*}, m^{*}\right)$ is locally indeterminate if and only if the elasticity of the money demand satisfies $\epsilon_{m m}<(\mu-\beta) / 2 \mu$ and one of the following conditions is satisfied:

i) the investment good is capital intensive around $\left(k^{*}, m^{*}\right)$;

ii) the consumption good is capital intensive around $\left(k^{*}, m^{*}\right)$ with $b \in(-\infty,-1 /(2-\delta)] \cup[-\beta /(1+(1-\delta) \beta), 0] ;$

iii) the consumption good is capital intensive around $\left(k^{*}, m^{*}\right)$ with $b \in(-1 /(2-\delta),-\beta /[1+(1-\delta) \beta])$ and $\epsilon_{c c}<\bar{\epsilon}_{c c}$.

\footnotetext{
${ }^{12}$ Note that the normality Assumption 3 implies $\xi_{c c}-\xi_{x c} \leq 0$ and $\xi_{x x}-\xi_{c x} \leq 0$.
} 
The existence of local indeterminacy with the CIA timing is easily obtained as it does not require particular restriction on the capital intensity difference across sectors. Notice however that it requires a low enough interest rate elasticity of money demand as in the aggregate formulation. ${ }^{13}$

Let us now consider the CAM timing. Contrary to the previous timing, local indeterminacy is always ruled out.

Theorem 2. With the CAM timing, let Assumptions 1-4 hold. Then the modified golden rule $\left(k^{*}, z^{*}\right)$ is always locally determinate.

With additively separable preferences we obtain the same conclusion as in the case of aggregate models in which $b=0$ : with the CAM timing, the equilibrium is unique and endogenous fluctuations based on self-fulfilling prophecies cannot occur. Note however that within aggregate models, this conclusion holds for any utility function. We then inquire whether this conclusion still holds for non-separable specifications. We will show that this is not the case by considering two different standard specifications of preferences. $^{14}$

\subsection{GHH preferences}

Consider a Greenwood-Hercovitz-Huffman [18] (GHH) formulation such that

$$
u(c, q \mathcal{M})=U(c+G(q \mathcal{M}))
$$

with $U($.$) and G($.$) some increasing and strictly concave functions. Such$ a specification implies that the marginal rate of substitution between consumption and real money balances depends on the latter only as

$$
\frac{u_{2}(c, q \mathcal{M})}{u_{1}(c, q \mathcal{M})}=G^{\prime}(q \mathcal{M})
$$

Let us denote

$$
\epsilon_{x x}^{G}=-\left(\xi_{x x}^{G}\right)^{-1}=-\left(G^{\prime \prime}(x) x / G^{\prime}(x)\right)^{-1}>0
$$

the elasticity of the function $G(x)$. Using (22) and (23), we derive at the steady state

$$
G^{\prime}\left(m^{*}\right)=\frac{\mu-\beta}{\beta}
$$

with the CIA timing, or

$$
G^{\prime}\left(z^{*}\right)=\frac{\mu-\beta}{\mu}
$$

\footnotetext{
${ }^{13}$ Theorem 1 generalizes the conclusions obtained by Bosi, Magris and Venditti [9] in a two-sector economy with CIA constraint.

${ }^{14}$ See Carlstrom and Fuerst [11].
} 
with the CAM timing. Let $v=c^{*} / m^{*}$ denote the steady state velocity of money and $z^{*}=\mu m^{*}$. We easily get from (26) that $\xi_{c c}=\xi_{x c}$ for $x=m$ or $z$ and

$$
\xi_{c m}=\xi_{c c} \frac{\mu-\beta}{\beta v}, \xi_{m m}=\xi_{c c} \frac{\mu-\beta}{\beta v}+\xi_{m m}^{G}
$$

with the CIA timing, or

$$
\xi_{c z}=\xi_{c c} \frac{\mu-\beta}{v}, \xi_{z z}=\xi_{c c} \frac{\mu-\beta}{v}+\xi_{z z}^{G}
$$

with the CAM timing.

Consider first the CIA timing. We introduce the following critical value for the elasticity of intertemporal substitution in consumption:

$$
\bar{\epsilon}_{c c}=\frac{\beta \theta^{2}+\frac{\mu-\beta}{\beta v} \frac{\varepsilon_{r k}}{\varepsilon_{c k}} b[1+(1-\delta) b]}{\frac{\varepsilon_{k} k}{\varepsilon_{c k}} b[1+(1-\delta) b]}>0
$$

Considering a particular interval of values for the capital intensity difference across sectors $b$ we get:

Theorem 3. With the CIA timing, let Assumptions 1-3 hold, $b \in(-1 /(1-$ $\delta),-1 /(2-\delta))$ and consider $\bar{\epsilon}_{c c}$ as defined by (32). Then the modified golden rule $\left(k^{*}, m^{*}\right)$ is locally indeterminate if $\epsilon_{c c}<\bar{\epsilon}_{c c}$ and $\epsilon_{m m}^{G} \leq(\mu-\beta) / 2 \mu$.

It is worth noting that local indeterminacy can arise for a broader range of values for $b$ but complex additional restrictions on technologies involving the elasticities $\varepsilon_{c k}$ and $\varepsilon_{r k}$ are necessary. Therefore, as our aim is to provide the simplest sufficient conditions for local indeterminacy, we focus on a specific range of values for $b$. Note also that the condition on the interest rate elasticity of money demand is the same as in the case of additively separable preferences. It is worth pointing out finally that negative values for $b$ are compatible with recent empirical evidences. Building on aggregate Input-Output tables, Takahashi et al. [29] have shown that over the last 30 years the OECD countries have been characterized by a consumption good sector which is more capital intensive than the investment good sector.

Consider now the CAM timing. We introduce the following critical value for the elasticity of intertemporal substitution in consumption:

$$
\tilde{\epsilon}_{c c} \equiv \frac{2\left[\frac{\mu-\beta}{v(1-\delta)^{2}} \frac{\varepsilon_{r k}}{\varepsilon_{c k}}+\beta(1+\beta) \theta^{2}\left(2-\frac{\mu-\beta}{\beta} \xi_{z z}^{G}\right)\right]}{\frac{\varepsilon_{r k}}{\varepsilon_{c k}(1-\delta)^{2}}\left(2-\frac{\mu-\beta}{\beta} \xi_{z z}^{G}\right)}>0
$$

Again, our strategy is to focus on the simplest sufficient conditions for the occurrence of local indeterminacy. We then consider a specific set of values for the capital intensity difference across sectors $b$ and some condition on the elasticities $\varepsilon_{c k}$ and $\varepsilon_{r k}$, although more general conclusions could be obtained at the price of additional restrictions on the technologies. 
Assumption 5. $b \in(-1 /(1-\delta)-\epsilon,-1 /(1-\delta)+\epsilon)$ with $\epsilon>0$ small enough, and $\lim _{\delta \rightarrow 1} \frac{\varepsilon_{r k}}{\varepsilon_{c k}(1-\delta)^{2}}=-\infty$.

Note from the capital accumulation equation (9) that the first part of Assumption 5 implies that around the steady state only small fluctuations of the capital stock and thus prices are considered..$^{15}$

Theorem 4. With the CAM timing, let Assumptions 1-3 and 5 hold. Then there exist $\underline{\delta} \in(0,1)$ and $\tilde{\epsilon}_{c c}>0$ as defined by (33) such that the modified golden rule $\left(k^{*}, z^{*}\right)$ is locally indeterminate if $\delta \in(\underline{\delta}, 1)$ and $\epsilon_{c c}<\tilde{\epsilon}_{c c}$.

The conclusion provided by Theorem 4 is important as it shows that contrary to the aggregate formulation with $b=0$, there exists some non-separable specification for preferences which allows for the existence of local indeterminacy when the consumption good sector is sufficiently capital intensive with respect to the investment good sector. Theorem 4 therefore shows that uniqueness no longer holds with the CAM timing if two sectors which are sufficiently asymmetric are considered.

\subsection{KPR preferences}

Consider a King-Plosser-Rebelo [21] (KPR) formulation such that

$$
u(c, x)=\frac{[c g(x)]^{1-\phi}}{1-\phi}
$$

which is compatible with balanced growth. Let us define $h(x)=g^{\prime}(x) / g(x)$ and

$$
\psi(x)=x h(x), \quad \eta(x)=\frac{x h^{\prime}(x)}{h(x)}
$$

Beside Assumption 3, we introduce the following restrictions:

Assumption 6. $g(x)$ is a positive increasing function, $\phi \geq 0, \eta(x) \leq$ $-\psi(x)(1-\phi)$ and $\eta(x) \leq \psi(x)(1-1 / \phi)$. Moreover $\lim _{x \rightarrow 0} h(x)=+\infty$ and $\lim _{x \rightarrow+\infty} h(x)=0$

Assumption 6 implies that Assumption 2 holds. ${ }^{16}$ Note that $\psi(x)>0$ can be interpreted as the elasticity of the utility of real money balances and $\eta(x)<0$ is linked to the interest rate elasticity of real money demand.

Using (22) and (23) and considering that $z^{*}=\mu m^{*}$ and $v^{*}=c^{*} / m^{*}$, we derive at the steady state

$$
\psi\left(m^{*}\right)=\frac{\mu-\beta}{\beta v^{*}}
$$

\footnotetext{
${ }^{15}$ We have indeed under Assumption 5: $d k_{t+1} / d k_{t}=1-\delta+d y_{t} / d k_{t}=1-\delta+1 / b \approx 0$.

${ }^{16}$ See Hintermaier [19, 20] and Pintus [25].
} 
with the CIA timing, or

$$
\psi\left(z^{*}\right)=\frac{\mu-\beta}{v^{*}}
$$

with the CAM timing. We then easily get from (26) that $\xi_{c c}=-\phi, \xi_{x c}=$ $1+\xi_{c c}$ for $x=m$ or $z$ and

$$
\xi_{c m}=\left(1+\xi_{c c}\right) \frac{\mu-\beta}{\beta v^{*}}, \xi_{m m}=\xi_{c m}+\eta\left(m^{*}\right)
$$

with the CIA timing, or

$$
\xi_{c z}=\left(1+\xi_{c c}\right) \frac{\mu-\beta}{v^{*}}, \xi_{z z}=\xi_{c z}+\eta\left(z^{*}\right)
$$

with the CAM timing.

Consider first the CIA timing and let $\eta=\eta\left(m^{*}\right)$. We proceed as in the case of GHH preferences and focus on a particular interval of values for $b$.

Theorem 5. With the CIA timing, let Assumptions 1-3 and 6 hold, $b \in$ $(-1 /(1-\delta),-1 /(2-\delta))$ and $\eta \leq-2 \mu /(\mu-\beta)$. Then there exists $\bar{\epsilon}_{c c}<0$ such that the modified golden rule $\left(k^{*}, m^{*}\right)$ is locally indeterminate if $\epsilon_{c c}<\bar{\epsilon}_{c c}$.

Again it is worth to mention that local indeterminacy can arise for a broader range of values of $b$ but complex additional restrictions on technologies involving the elasticities $\varepsilon_{c k}$ and $\varepsilon_{r k}$ are necessary. Note also that the condition on the elasticity $\eta$ is the same as in the cases of additively separable and GHH preferences since it implies to consider a low enough interest rate elasticity of real money demand.

Consider now the CAM timing and let $\eta=\eta\left(z^{*}\right)$. We introduce the following critical value for the elasticity of intertemporal substitution in consumption:

$$
\tilde{\epsilon}_{c c} \equiv \min \left\{\frac{\beta-(\mu-\beta)\left(\eta-\frac{\mu-\beta}{v^{*}}\right)}{\frac{(\mu-\beta)^{2}}{v^{*}}}, \frac{2 \frac{\mu-\beta}{v^{*}}}{2+\frac{\mu-\beta}{v^{*}}\left(2-\frac{\eta v^{*}}{\beta}\right)}\right\}>0
$$

We also proceed as in the case of GHH preferences and consider a specific set of values for $b$ which is far enough from zero and which implies that around the steady state, the fluctuations of prices are small.

Theorem 6. With the CAM timing, let Assumptions 1-3, 5 and 6 hold. Then there exist $\underline{\delta} \in(0,1)$ and $\tilde{\epsilon}_{c c}>0$ as defined by (38) such that the modified golden rule $\left(k^{*}, z^{*}\right)$ is locally indeterminate if $\delta \in(\underline{\delta}, 1)$ and $\epsilon_{c c}=$ $1 / \phi<\tilde{\epsilon}_{c c}$.

Theorem 6 confirms the conclusion given by Theorem 4 in the case of a GHH utility function: it proves that there exist standard non-separable 
specifications of preferences for which local indeterminacy arises with both formulations of the timing for money. These results are based on a capital intensity difference across sectors which is sufficiently negative and show that two-sector models lead to a greater flexibility in terms of local indeterminacy of equilibrium paths than aggregate models.

\section{Example}

We do not consider additively separable preferences as we have shown that the main conclusions are similar to those obtained with an aggregate formulation. Moreover, we focus on the CAM timing as the proof of existence of local indeterminacy in this case is the main contribution of our paper.

Assume that preferences are given either by a GHH utility function

$$
u^{G H H}(c, x)=\frac{1}{1-\varphi}\left(c+\zeta \frac{x^{1-\nu}}{1-\nu}\right)^{1-\varphi}
$$

or a KPR utility function

$$
u^{K P R}(c, x)=\frac{1}{1-\phi}\left(c \frac{x^{1+\rho}}{1+\rho}\right)^{1-\phi}
$$

with $\varphi, \nu, \phi \geq 0, \rho>-1$ and $\zeta>0$. Consider also the following CES technologies:

$$
\begin{aligned}
& f^{0}\left(k_{0}, l_{0}\right)=\left[\chi k_{0}^{-\sigma}+(1-\chi) l_{0}^{-\sigma}\right]^{-1 / \sigma}=y_{0} \\
& f^{1}\left(k_{1}, l_{1}\right)=\min \left\{k_{1} / \gamma, l_{1}\right\}=y
\end{aligned}
$$

with $\chi \in(0,1), \sigma>-1$ and $\gamma>0$. Since at the equilibrium $y=k_{1} / \gamma=l_{1}$, we get $k_{0}=k-\gamma y, l_{0}=1-y$ and thus the social production function

$$
T(k, y)=\left[\chi(k-\gamma y)^{-\sigma}+(1-\chi)(1-y)^{-\sigma}\right]^{-1 / \sigma}
$$

Assuming that $\beta \theta-\gamma>0$, the steady state for capital is

$$
k^{*}=\left[\left(\frac{1-\chi}{\chi} \frac{1}{\beta \theta-\gamma}\right)^{1 /(1+\sigma)}(1-\gamma \delta)+\delta\right]^{-1}
$$

and since $y^{*}=\delta k^{*}$, we easily derive

$$
\begin{aligned}
\frac{\varepsilon_{r k}^{*}}{\varepsilon_{c k}^{*}} & =-(1+\sigma)\left(\frac{1-\chi}{\chi}\right)^{1 /(1+\sigma)}(\beta \theta-\gamma)^{\sigma /(1+\sigma)}<0 \\
b & =\gamma-\left[\frac{\chi(\beta \theta-\gamma)}{1-\chi}\right]^{1 /(1+\sigma)}
\end{aligned}
$$

As a result we have $b=-1 /(1-\delta)$ if and only if

$$
\left(\frac{1-\chi}{\chi} \frac{1}{\beta \theta-\gamma}\right)^{1 /(1+\sigma)}=\frac{1-\delta}{1+\gamma(1-\delta)}
$$


Obviously, the previous equality holds if $\chi=\hat{\chi}$ as given by

$$
\hat{\chi}=\left[1+(\beta \theta-\gamma)\left(\frac{1-\delta}{1+\gamma(1-\delta)}\right)^{1+\sigma}\right]^{-1}
$$

Moreover, when $\chi=\hat{\chi}$ we have

$$
\left(\frac{1-\hat{\chi}}{\hat{\chi}}\right)^{1 /(1+\sigma)}=\frac{(1-\delta)(\beta \theta-\gamma)^{1 /(1+\sigma)}}{1+\gamma(1-\delta)}
$$

and thus we get

$$
\frac{\varepsilon_{r k}^{*}}{\varepsilon_{c k}^{*}}=-(1+\sigma) \frac{(1-\delta)(\beta \theta-\gamma)}{1+\gamma(1-\delta)}
$$

so that

$$
\frac{\varepsilon_{r k}}{\varepsilon_{c k}(1-\delta)^{2}}=-\frac{(1+\sigma)(\beta \theta-\gamma)}{(1-\delta)[1+\gamma(1-\delta)]}
$$

As $\theta \in[0,1]$ for any $\delta \in[0,1]$, Assumption 5 is satisfied. We also derive $k^{*}=1+\gamma(1-\delta)$ which leads to

$$
c^{*}=T\left(k^{*}, \delta k^{*}\right)=(1-\gamma \delta)\left[\frac{[1+\gamma(1-\delta)]^{1+\sigma}+(\beta \theta-\gamma)(1-\delta)^{1+\sigma}}{\theta}\right]^{1 / \sigma}
$$

Consider first the case of GHH preferences. Solving the first order condition (23) using (39) gives at the steady state

$$
z^{*}=\mu m^{*}=\left(\frac{\mu-\beta}{\zeta \mu}\right)^{-1 / \nu}
$$

and we derive from (41) the stationary velocity of money

$$
v^{*} \equiv c^{*} / m^{*}=\mu\left(\frac{\mu-\beta}{\zeta \mu}\right)^{1 / \nu}(1-\gamma \delta)\left[\frac{[1+\gamma(1-\delta)]^{1+\sigma}+(\beta \theta-\gamma)(1-\delta)^{1+\sigma}}{\theta}\right]^{1 / \sigma}
$$

It follows from (39) that

$$
\epsilon_{c c}=\frac{1}{\varphi}+\frac{\mu-\beta}{\varphi(1-\nu) v^{*}}
$$

Moreover, as $\xi_{x x}^{G}=-\nu$ for all $x>0$, we derive from (33) the following critical value for the elasticity of intertemporal substitution in consumption:

$$
\tilde{\epsilon}_{c c} \equiv 2 \beta\left[\frac{\mu-\beta}{v^{*}[2 \beta+(\mu-\beta) \nu]}-\frac{(1+\beta) \theta^{2}(1-\delta)[1+\gamma(1-\delta)]}{(1+\sigma)(\beta \theta-\gamma)}\right]
$$

The critical bound $\underline{\delta} \in[0,1)$ is obtained as the value of $\delta$ such that the expression between brackets on the right-hand-side is greater or equal to zero. ${ }^{17}$ Assuming $\delta \in(\underline{\delta}, 1)$, we conclude that there exists $\underline{\varphi}>0$ such that

\footnotetext{
${ }^{17}$ Note that $\underline{\delta}=0$ if

$$
\frac{\mu-\beta}{2 \beta+(\mu-\beta) \nu} \geq \frac{(1+\beta)(1+\gamma) \mu\left(\frac{\mu-\beta}{\zeta \mu}\right)^{1 / \nu}\left[(1-\beta)\left[(1+\gamma)^{1+\sigma}-\gamma\right]+\beta\right]^{1 / \sigma}}{(1+\sigma)(1-\beta)[\beta-\gamma(1-\beta)]}
$$
}


$\epsilon_{c c}<\tilde{\epsilon}_{c c}$, and thus local indeterminacy arises, if $\varphi>\underline{\varphi}$.

Consider now the case of KPR preferences. We derive from (40) that $\psi(x)=1+\rho$ and $\eta(x)=-1$ for all $x>0$. Assumption 6 then requires $1 \geq(1+\rho)(1-\phi)$ and $1 \geq(1+\rho)(1-\phi) / \phi$. Solving the first order condition (23) with $z=\mu m$ gives the stationary velocity of money

$$
v^{*} \equiv c^{*} / m^{*}=\frac{\mu-\beta}{1+\rho}
$$

Using (38) we derive the following critical value for the elasticity of intertemporal substitution in consumption:

$$
\tilde{\epsilon}_{c c} \equiv \frac{2 \beta(1+\rho)}{\mu+\beta+2 \beta(1+\rho)}
$$

Therefore, there exists $\phi>0$ such that Assumption 6 holds and

$$
\epsilon_{c c} \equiv \frac{1}{\phi}<\tilde{\epsilon}_{c c}
$$

if $\phi>\phi$. Under this restriction we derive the critical bound $\underline{\delta} \in(0,1)$ as the value of $\delta$ such that the following equation is satisfied

$$
\frac{\beta \theta-\gamma}{(1-\delta)[1+\gamma(1-\delta)] \beta \theta^{2}}=2 \frac{1+\beta}{1+\sigma} \frac{(1-\phi)(1+\rho) \frac{\mu-\beta}{\mu+\beta}-\phi}{(1-\phi)(1+\rho) \frac{2 \beta}{\mu+\beta}+1}
$$

We then conclude that local indeterminacy arises if $\phi>\phi$ and $\delta \in(\underline{\delta}, 1)$.

\section{Concluding comments}

We have considered a two-sector MIUF model with general technologies and preferences defined on the basis of three popular specifications of the utility function in the business cycle and growth literature: i) an additively separable formulation, ii) a KPR formulation, and iii) a GHH formulation. The CIA and CAM timings have been systematically compared throughout the paper. First, we have shown that for all the three different specifications of preferences, local indeterminacy with a CIA timing requires a low enough interest rate elasticity of money demand. Second, and contrary to the aggregate formulation, we have proved the possible existence of local indeterminacy with a CAM timing depending on the utility function. While we have shown that determinacy always holds under separable preferences, we have provided simple sufficient conditions for local indeterminacy in the case of GHH [18] and KPR [21] preferences. Explicit examples with CES technologies have also been given to illustrate these theoretical findings. We have thus proved that compared to aggregate models, two-sector models provide new rooms for local indeterminacy when non-separable standard preferences are considered. 


\section{Appendix}

\subsection{Proof of Proposition 1}

Consider in a first step equation (21). Note that the steady state value of $k$ only depends on the characteristics of the technologies and is independent of the per-period utility function. The proof of Theorem 3.1 in Becker and Tsyganov [3] restricted to the case of one homogeneous agent applies so that there exists a unique $k^{*}$ solution of $(21)$.

Consider in a second step equations (22) and (23) evaluated at $k^{*}$. We then write these two conditions as:

$$
\frac{u_{2}\left(T\left(k^{*}, \delta k^{*}\right), x\right)}{u_{1}\left(T\left(k^{*}, \delta k^{*}\right), x\right)} \equiv \Theta(x)=\pi
$$

with $(x, \pi)=(m, \mu / \beta-1)$ or $(z, 1-\beta / \mu)$. The function $\Theta(x)$ satisfies

$$
\Theta^{\prime}(x)=\frac{u_{22} u_{1}-u_{12} u_{2}}{u_{1}^{2}}
$$

The normality Assumption 3 implies that for any $x>0, \Theta^{\prime}(x)<0$. This monotonicity property together with the boundary conditions in Assumption 2 finally guarantee the existence and uniqueness of a solution $m^{*}$ or $z^{*}$ of equation (22) or (23).

\subsection{The characteristic polynomial}

In order to derive a tractable formulation for the third-degree polynomial associated with the dynamical system (17), we need to compute the second derivatives of $T(k, y)$. Let $b$ be the capital intensity difference across sectors as defined by (24). We know from Benhabib and Nishimura [5] that

$$
T_{12}=-T_{11} b, \quad T_{22}=T_{11} b^{2}
$$

with $T_{11}<0$ implied by the concavity of $T(k, y)$.

As shown previously, the steady states $\left(k^{*}, m^{*}\right)$ and $\left(k^{*}, z^{*}\right)$ are characterized by equations (21)-(23) which can be written as follows

$$
\begin{aligned}
-T_{2}^{*} \beta^{-1} & =T_{1}^{*}-(1-\delta) T_{2}^{*} \\
u_{1}^{m} & =\frac{\beta}{\mu}\left(u_{1}^{m}+u_{2}^{m}\right) \\
\frac{\beta}{\mu} u_{1}^{z} & =u_{1}^{z}-u_{2}^{z}
\end{aligned}
$$

with $T_{i}^{*}=T_{i}\left(k^{*}, \delta k^{*}\right), u_{i}^{m}=u_{i}\left(c^{*}, m^{*}\right)$ and $u_{i}^{z}=u_{i}\left(c^{*}, z^{*}\right)$. In the following we will also consider a second formulation for equation (43)

$$
-T_{2}^{*}=\beta \theta T_{1}^{*}
$$


with $\theta=[1-\beta(1-\delta)]^{-1}$. The second derivatives of the function $T(k, y)$ will be evaluated at $k^{*}$ using the following notation: $T_{i j}^{*}=T_{i j}\left(k^{*}, \delta k^{*}\right)$.

Using equations (25)-(27) and (42)-(46), and denoting $d \kappa_{t}=d k_{t+1}$, total differenciation of the Euler equations (17) gives after tedious but straightforward computations the following linear system

$$
\begin{aligned}
\left(\begin{array}{ccc}
0 & -\beta \mathcal{A}_{1}^{m} & \mathcal{A}_{2}^{m} \\
0 & \beta \mathcal{A}_{3}^{m} & \mathcal{A}_{4}^{m} \\
1 & 0 & 0
\end{array}\right)\left(\begin{array}{c}
d k_{t+1} \\
d \kappa_{t+1} \\
d m_{t+1}
\end{array}\right) \\
=\left(\begin{array}{ccc}
\mathcal{A}_{1}^{m} & \mathcal{A}_{5}^{m} & \mathcal{A}_{2}^{m} \\
-\xi_{c c} \theta & \xi_{c c} \beta \theta+\mathcal{A}_{3}^{m} & -\frac{c^{*}}{m^{*} T_{1}^{*}}\left(1+\xi_{c m}\right) \\
0 & 1 & 0
\end{array}\right)\left(\begin{array}{c}
d k_{t} \\
d \kappa_{t} \\
d m_{t}
\end{array}\right)
\end{aligned}
$$

where

$$
\begin{aligned}
\mathcal{A}_{1}^{m} & =\beta \theta^{2} \xi_{c c}+\frac{\varepsilon_{r k}}{\varepsilon_{c k}} b[1+(1-\delta) b] \\
\mathcal{A}_{2}^{m} & =\frac{\theta}{T_{1}^{*}} \xi_{m c}(\mu-\beta) \\
\mathcal{A}_{3}^{m} & =\frac{\theta}{\mu}\left[\xi_{c c} \beta+\xi_{m c}(\mu-\beta)\right] \\
\mathcal{A}_{4}^{m} & =-\frac{c^{*}}{m^{*} T_{1}^{*}}\left[1+\frac{1}{\mu}\left(\xi_{c m} \beta+\xi_{m m}(\mu-\beta)\right)\right] \\
\mathcal{A}_{5}^{m} & =-\beta(1+\beta) \theta^{2} \xi_{c c}-\frac{\varepsilon_{r k}}{\varepsilon_{c k}}\left[b^{2}+\beta[1+(1-\delta) b]^{2}\right]>0
\end{aligned}
$$

with the CIA timing, or

$$
\left(\begin{array}{ccc}
\mathcal{A}_{1}^{z} & -\beta \mathcal{A}_{2}^{z} & \frac{\xi_{c z}}{\theta \varepsilon_{c k}} \\
\xi_{c c} & -\beta \xi_{c c} & \frac{1+\xi_{c z}}{\theta \varepsilon_{c k}} \\
1 & 0 & 0
\end{array}\right)\left(\begin{array}{c}
d k_{t+1} / k \\
d \kappa_{t+1} / \kappa \\
d z_{t+1} / z
\end{array}\right)=\left(\begin{array}{ccc}
\mathcal{A}_{2}^{z} & -\beta \mathcal{A}_{3}^{z} & \frac{\xi_{c z}}{\theta \varepsilon_{c k}} \\
\mathcal{A}_{4}^{z} & -\beta \mathcal{A}_{4}^{z} & \frac{1+\mathcal{A}_{5}^{z}}{\theta \varepsilon_{c k}} \\
0 & 1 & 0
\end{array}\right)\left(\begin{array}{c}
d k_{t} / k \\
d \kappa_{t} / \kappa \\
d z_{t} / z
\end{array}\right)
$$

where

$$
\begin{aligned}
\mathcal{A}_{1}^{z} & =\xi_{c c}+\frac{\varepsilon_{r k}}{\varepsilon_{c k}}\left[\frac{1+(1-\delta) b}{\theta}\right]^{2}<0 \\
\mathcal{A}_{2}^{z} & =\xi_{c c}+\frac{\varepsilon_{r k}}{\varepsilon_{c k}} \frac{b}{\beta \theta} \frac{1+(1-\delta) b}{\theta} \\
\mathcal{A}_{3}^{z} & =\xi_{c c}+\frac{\varepsilon_{r k}}{\varepsilon_{c k}}\left(\frac{b}{\beta \theta}\right)^{2}<0 \\
\mathcal{A}_{4}^{z} & =\frac{\mu}{\beta} \xi_{c c}+\left(1-\frac{\mu}{\beta}\right) \xi_{z c} \\
\mathcal{A}_{5}^{z} & =\frac{\mu}{\beta} \xi_{c z}+\left(1-\frac{\mu}{\beta}\right) \xi_{z z}
\end{aligned}
$$

with the CAM timing. 
Assuming that with both timings the matrix on the left-hand-side is nonsingular, the following lemma provides a formulation for the characteristic polynomial:

Lemma 1. Under Assumptions 1-3, let $\mathcal{A}_{1}^{m} \mathcal{A}_{4}^{m}+\mathcal{A}_{2}^{m} \mathcal{A}_{3}^{m} \neq 0$ and $\mathcal{A}_{2}^{z}\left(1+\xi_{c z}\right)-$ $\xi_{c c} \xi_{c z} \neq 0$. The characteristic polynomial is

where

$$
\mathcal{P}(\lambda)=\lambda^{3}-\mathcal{T} \lambda^{2}+\mathcal{S} \lambda-\mathcal{D}
$$

$$
\begin{aligned}
\mathcal{D} & =\frac{\mathcal{A}_{2}^{m} \xi_{c c} \theta-\frac{\mathcal{A}_{1}^{m} c^{*}}{T_{1}^{*} m^{*}}\left(1+\xi_{c m}\right)}{\beta\left(\mathcal{A}_{1}^{m} \mathcal{A}_{4}^{m}+\mathcal{A}_{2}^{m} \mathcal{A}_{3}^{m}\right)} \\
\mathcal{S} & =\frac{1}{\beta}+\frac{\mathcal{A}_{2}^{m} \xi_{c c} \theta(1+\beta)+\frac{\mathcal{A}_{5}^{m} c^{*}}{T_{1}^{*} m^{*}}\left(1+\xi_{c m}\right)}{\beta\left(\mathcal{A}_{1}^{m} \mathcal{A}_{4}^{m}+\mathcal{A}_{2}^{m} \mathcal{A}_{3}^{m}\right)} \\
\mathcal{T} & =\beta \mathcal{D}+\frac{\mathcal{A}_{2}^{m} \mathcal{A}_{3}^{m}(1+\beta)-\mathcal{A}_{4}^{m} \mathcal{A}_{5}^{m}}{\beta\left(\mathcal{A}_{1}^{m} \mathcal{A}_{4}^{m}+\mathcal{A}_{2}^{m} \mathcal{A}_{3}^{m}\right)}
\end{aligned}
$$

with the CIA timing, or

$$
\begin{aligned}
\mathcal{D} & =\frac{\mathcal{A}_{2}^{z}\left(1+\mathcal{A}_{5}\right)-\mathcal{A}_{4}^{z} \xi_{c z}}{\beta\left[\mathcal{A}_{2}^{z}\left(1+\xi_{c z}\right)-\xi_{c c} \xi_{c z}\right]} \\
\mathcal{S} & =\frac{1}{\beta}+\frac{\left(\beta \mathcal{A}_{3}^{z}+\mathcal{A}_{1}^{z}\right)\left(1+\mathcal{A}_{5}^{z}\right)-\mathcal{A}_{4}^{z} \xi_{c z}(1+\beta)}{\beta\left[\mathcal{A}_{2}^{z}\left(1+\xi_{c z}\right)-\xi_{c c} \xi_{c z}\right]} \\
\mathcal{T} & =\beta \mathcal{D}+\frac{\left(\beta \mathcal{A}_{3}^{z}+\mathcal{A}_{1}^{z}\right)\left(1+\xi_{c z}\right)-\xi_{c c} \xi_{c z}(1+\beta)}{\beta\left[\mathcal{A}_{2}^{z}\left(1+\xi_{c z}\right)-\xi_{c c} \xi_{c z}\right]}
\end{aligned}
$$

with the CAM timing.

Proof: Considering that $\xi_{c m} \beta\left(c^{*} / m^{*}\right)=\xi_{m c}(\mu-\beta)$ and $\xi_{c z} \mu\left(c^{*} / z^{*}\right)=$ $\xi_{z c}(\mu-\beta)$, tedious but straightforward computations give the result.

In the sequel, the local stability analysis will be derived considering the characteristic polynomial evaluated at $\lambda=1, \lambda=0$ and $\lambda=-1$. We easily compute from Lemma 1:

$$
\begin{aligned}
\frac{\mathcal{P}(1)}{\mathcal{P}(0)} & =\frac{\mu-\beta}{\mu} \frac{\varepsilon_{r k}}{\frac{\varepsilon}{c k}^{2}}(1-\delta b)\left(\beta-\theta^{-1} b\right)\left(\xi_{m m}-\xi_{c m}\right) \\
\frac{\mathcal{P}(-1)}{\mathcal{P}(0)} & =\frac{\frac{\varepsilon_{r k}}{\varepsilon_{c k}}[1+(2-\delta) b][\beta+b[1+(1-\delta) \beta]]\left[2+\frac{1}{\mu}\left[\xi_{c m}(\mu+\beta)+\xi_{m m}(\mu-\beta)\right]\right]}{\beta \theta^{2} \xi_{c c}+\left(1+\xi_{c m}\right) \frac{\varepsilon_{r k}}{\varepsilon_{c k}} b[1+(1-\delta) b]} \\
& +\frac{2 \beta(1+\beta) \theta^{2}\left[2 \xi_{c c}+\frac{\mu-\beta}{\mu}\left(\xi_{c c} \xi_{m m}-\xi_{c m} \xi_{m c}\right)\right]}{\beta \theta^{2} \xi_{c c}+\left(1+\xi_{c m}\right) \frac{\varepsilon_{r k}}{\varepsilon_{c k}} b[1+(1-\delta) b]}
\end{aligned}
$$

with the CIA timing, and 


$$
\begin{aligned}
\frac{\mathcal{P}(1)}{\mathcal{P}(0)} & =\frac{\frac{\mu-\beta}{\beta}\left(\xi_{z z}-\xi_{c z}\right) \frac{(1-\delta b)\left(\beta-\theta^{-1} b\right)}{\beta \theta^{2}} \frac{\varepsilon_{r k}}{\varepsilon_{c k}}}{\xi_{c c}+\left(1+\xi_{c z}\right) \frac{1+(1-\delta) b}{\theta} \frac{b}{\beta \theta} \frac{\varepsilon_{r k}}{\varepsilon_{c k}}-\frac{\mu-\beta}{\beta}\left[\xi_{c c} \xi_{z z}-\xi_{c z} \xi_{z c}+\left(\xi_{z z}-\xi_{c z}\right) \frac{1+(1-\delta) b}{\theta} \frac{b}{\beta \theta} \frac{\varepsilon_{r k}}{\varepsilon_{c k}}\right]} \\
\frac{\mathcal{P}(-1)}{\mathcal{P}(0)} & =\frac{\left[2\left(1+\xi_{c z}\right)-\frac{\mu-\beta}{\beta}\left(\xi_{z z}-\xi_{c z}\right)\right]\left[\frac{1+(1-\delta) b}{\theta}+\frac{b}{\theta}\right]\left[\frac{1+(1-\delta) b}{\theta}+\frac{b}{\beta \theta}\right] \frac{\varepsilon_{r k}}{\varepsilon_{c k}}}{\xi_{c c}+\left(1+\xi_{c z}\right) \frac{1+(1-\delta) b}{\theta} \frac{b}{\beta \theta} \frac{\varepsilon_{r k}}{\varepsilon_{c k}}-\frac{\mu-\beta}{\beta}\left[\xi_{c c} \xi_{z z}-\xi_{c z} \xi_{z c}+\left(\xi_{z z}-\xi_{c z}\right) \frac{1+(1-\delta) b}{\theta} \frac{b}{\beta \theta} \frac{\varepsilon_{r k}}{\varepsilon_{c k}}\right]} \\
& +\frac{2(1+\beta)\left[2 \xi_{c c}-\frac{\mu-\beta}{\beta}\left(\xi_{c c} \xi_{z z}-\xi_{c z} \xi_{z c}\right)\right]}{\xi_{c c}+\left(1+\xi_{c z}\right) \frac{1+(1-\delta) b}{\theta} \frac{b}{\beta \theta} \frac{\varepsilon_{r k}}{\varepsilon_{c k}}-\frac{\mu-\beta}{\beta}\left[\xi_{c c} \xi_{z z}-\xi_{c z} \xi_{z c}+\left(\xi_{z z}-\xi_{c z}\right) \frac{1+(1-\delta) b}{\theta} \frac{b}{\beta \theta} \frac{\varepsilon_{r k}}{\varepsilon_{c k}}\right]}
\end{aligned}
$$

with the CAM timing.

\subsection{Proof of Theorem 1}

Under Assumption 4 we derive from Lemma 1 that

$$
\mathcal{D}=\frac{1}{\beta\left[1+\xi_{m m} \frac{\mu-\beta}{\mu}\right]}, \quad \mathcal{S}=\frac{1}{\beta}-\frac{\mathcal{A}_{5}^{m}}{\mathcal{A}_{1}^{m}} \mathcal{D}, \quad \mathcal{T}=\beta \mathcal{D}-\frac{\mathcal{A}_{5}^{m}}{\beta \mathcal{A}_{1}^{m}}
$$

and thus the characteristic polynomial rewrites as

$$
\begin{aligned}
\mathcal{P}(\lambda) & =\left(\lambda^{2}+\lambda \frac{\mathcal{A}_{5}^{m}}{\beta \mathcal{A}_{1}^{m}}+\frac{1}{\beta}\right)(\lambda-\beta \mathcal{D}) \\
& =\left(\lambda^{2}-\lambda \frac{\beta(1+\beta) \theta^{2} \xi_{c c}+\frac{\varepsilon_{r k}}{\varepsilon_{c k}}\left[b^{2}+\beta[1+(1-\delta) b]^{2}\right]}{\beta\left[\beta \theta^{2} \xi_{c c}+\frac{\varepsilon_{k}}{\varepsilon_{c k}} b[1+(1-\delta) b]\right]}+\frac{1}{\beta}\right)(\lambda-\beta \mathcal{D}) \\
& \equiv \tilde{\mathcal{P}}(\lambda)(\lambda-\beta \mathcal{D})
\end{aligned}
$$

Therefore, when the utility is additively separable, the characteristic roots are separated. Note that $\tilde{\mathcal{P}}(\lambda)$ corresponds to the second-degree characteristic polynomial obtained within a standard two-sector optimal growth model without money (see Bosi, Magris and Venditti [8]). It follows that one characteristic root is within the unit circle while the other is outside in the following cases:

i) $b \in(-\infty,-1 /(2-\delta)] \cup[-\beta /(1+(1-\delta) \beta), \beta \theta)$,

ii) $b \in(-1 /(2-\delta),-\beta /[1+(1-\delta) \beta])$ and

$$
\xi_{c c}<-\frac{\frac{\varepsilon_{r k}}{\varepsilon_{c k}}[1+(2-\delta) b][\beta+b[1+(1-\delta) \beta]]}{2 \beta(1+\beta) \theta^{2}} \equiv \bar{\xi}_{c c}
$$

The third characteristic root is obviously derived from (50) and depends on money as

$$
\lambda=\beta \mathcal{D}=\frac{1}{1+\xi_{m m} \frac{\mu-\beta}{\mu}}
$$

Therefore, $\lambda \in(-1,0)$ if and only if $\xi_{m m}<-2 \mu /(\mu-\beta)$. Let $\bar{\epsilon}_{c c}=-\bar{\xi}_{c c}^{-1}$ and $\bar{\epsilon}_{m m}=(\mu-\beta) /(2 \mu)$. The final results follow from (25) and (26). 


\subsection{Proof of Theorem 2}

Under Assumption 4 we derive from Lemma 1 that

$$
\mathcal{D}=\frac{1-\left(\frac{\mu-\beta}{\beta}\right) \xi_{z z}}{\beta}, \quad \mathcal{S}=\frac{1}{\beta}+\beta \mathcal{D}\left(\frac{\mathcal{A}_{1}^{z}}{\beta \mathcal{A}_{2}^{z}}+\frac{\mathcal{A}_{3}^{z}}{\mathcal{A}_{2}^{z}}\right), \quad \mathcal{T}=\beta \mathcal{D}+\frac{\mathcal{A}_{1}^{z}}{\beta \mathcal{A}_{2}^{z}}+\frac{\mathcal{A}_{3}^{z}}{\mathcal{A}_{2}^{z}}
$$

and thus the characteristic polynomial rewrites as

$$
\begin{aligned}
\mathcal{P}(\lambda) & =\left(\lambda^{2}-\lambda\left[\frac{\mathcal{A}_{1}^{z}}{\beta \mathcal{A}_{2}^{z}}+\frac{\mathcal{A}_{3}^{z}}{\mathcal{A}_{2}^{z}}\right]+\frac{1}{\beta}\right)(\lambda-\beta \mathcal{D}) \\
& =\tilde{\mathcal{P}}(\lambda)(\lambda-\beta \mathcal{D})
\end{aligned}
$$

with $\tilde{\mathcal{P}}(\lambda)$ as defined by (50). As in the CIA timing the characteristic roots are separated, and the first two characteristic roots have the same properties as in the proof of Theorem 1 . The third root satisfies

$$
\lambda=\beta \mathcal{D}=1-\frac{\mu-\beta}{\beta} \xi_{z z}>1
$$

The result follows from (26).

\subsection{Proof of Theorem 3}

When the utility function assumes the GHH formulation, we have with the CIA timing

$$
\xi_{c c}=\xi_{m c}, \xi_{c m}=\xi_{c c} \frac{\mu-\beta}{\beta v}, \xi_{m m}=\xi_{c c} \frac{\mu-\beta}{\beta v}+\xi_{m m}^{G}
$$

It follows from (48) that

$$
\begin{aligned}
\frac{\mathcal{P}(1)}{\mathcal{P}(0)} & =\frac{\mu-\beta}{\mu} \frac{\frac{\varepsilon_{r k}}{\varepsilon_{c k}}(1-\delta b)\left(\beta-\theta^{-1} b\right) \xi_{m m}^{G}}{\xi_{c c}\left(\beta \theta^{2}+\frac{\mu-\beta}{\beta v} \frac{\varepsilon_{r k}}{\varepsilon_{c k}} b[1+(1-\delta) b]\right)+\frac{\varepsilon_{r k}}{\varepsilon_{c k}} b[1+(1-\delta) b]} \\
\frac{\mathcal{P}(-1)}{\mathcal{P}(0)} & =\frac{2 \xi_{c c}\left[\frac{\mu-\beta}{\beta v} \frac{\varepsilon_{r k}}{\varepsilon_{c k}}[1+(2-\delta) b][\beta+b[1+(1-\delta) \beta]]+\beta(1+\beta) \theta^{2}\left(2+\frac{\mu-\beta}{\mu} \xi_{m m}^{G}\right)\right]}{\xi_{c c}\left(\beta \theta^{2}+\frac{\mu-\beta}{\beta v} \frac{\varepsilon_{r k}}{\varepsilon_{c k}} b[1+(1-\delta) b]\right)+\frac{\varepsilon_{r k}}{\varepsilon_{c k}} b[1+(1-\delta) b]} \\
& +\frac{\frac{\varepsilon_{r k}}{\varepsilon_{c k}}[1+(2-\delta) b][\beta+b[1+(1-\delta) \beta]]\left(2+\frac{\mu-\beta}{\mu} \xi_{m m}^{G}\right)}{\xi_{c c}\left(\beta \theta^{2}+\frac{\mu-\beta}{\beta v} \frac{\varepsilon_{r k}}{\varepsilon_{c k}} b[1+(1-\delta) b]\right)+\frac{\varepsilon_{r k}}{\varepsilon_{c k}} b[1+(1-\delta) b]}
\end{aligned}
$$

Local indeterminacy will be obtained if $\mathcal{P}(1) / \mathcal{P}(0)<0$ and $\mathcal{P}(-1) / \mathcal{P}(0)<0$. Assume that $b \in(-1 /(1-\delta),-1 /(2-\delta))$, and consider first $\mathcal{P}(1) / \mathcal{P}(0)$. As the numerator is positive and $b[1+(1-\delta) b]<0$, the denominator is negative, and thus $\mathcal{P}(1) / \mathcal{P}(0)<0$, if

$$
\xi_{c c}<-\frac{\frac{\varepsilon_{r k}}{\varepsilon_{c k}} b[1+(1-\delta) b]}{\beta \theta^{2}+\frac{\mu-\beta}{\beta v} \frac{\varepsilon_{k} k}{\varepsilon_{c k}} b[1+(1-\delta) b]} \equiv \bar{\xi}_{c c}
$$


Consider then $\mathcal{P}(-1) / \mathcal{P}(0)$. As the denominator is negative, $\mathcal{P}(-1) / \mathcal{P}(0)<$ 0 if the numerator is positive. Since $[1+(2-\delta) b][\beta+b[1+(1-\delta) \beta]]>0$, we get a positive numerator if

$$
2+\frac{\mu-\beta}{\mu} \xi_{m m}^{G} \leq 0
$$

The result follows.

\subsection{Proof of Theorem 4}

When the utility function assumes the GHH formulation, we have with the CAM timing

$$
\xi_{c c}=\xi_{z c}, \quad \xi_{c z}=\xi_{c c} \frac{\mu-\beta}{v}, \xi_{z z}=\xi_{c c} \frac{\mu-\beta}{v}+\xi_{z z}^{G}
$$

It follows from (49) that

$$
\begin{aligned}
\frac{\mathcal{P}(1)}{\mathcal{P}(0)} & =\frac{\mu-\beta}{\beta} \frac{\frac{\varepsilon_{r k}}{\varepsilon_{c k}}(1-\delta b)\left(\beta-\theta^{-1} b\right) \xi_{z z}^{G}}{\xi_{c c}\left(\beta \theta^{2}+\frac{\mu-\beta}{v} \frac{\varepsilon_{r k}}{\varepsilon_{c k}} b[1+(1-\delta) b]-\frac{\mu-\beta}{\beta} \beta \theta^{2} \xi_{z z}^{G}\right)+\frac{\varepsilon_{r k}}{\varepsilon_{c k}} b[1+(1-\delta) b]\left(1-\frac{\mu-\beta}{\beta} \xi_{z z}^{G}\right)} \\
\frac{\mathcal{P}(-1)}{\mathcal{P}(0)} & =\frac{2 \xi_{c c}\left[\frac{\mu-\beta}{v} \frac{\varepsilon_{r k}}{\varepsilon_{k}}[1+(2-\delta) b][\beta+b[1+(1-\delta) \beta]]+\beta(1+\beta) \theta^{2}\left(2-\frac{\mu-\beta}{\beta} \xi_{z z}^{G}\right)\right]}{\xi_{c c}\left(\beta \theta^{2}+\frac{\mu-\beta}{v} \frac{\varepsilon_{r k}}{\varepsilon_{c k}} b[1+(1-\delta) b]-\frac{\mu-\beta}{\beta} \beta \theta^{2} \xi_{z z}^{G}\right)+\frac{\varepsilon_{r k}}{\varepsilon_{c k}} b[1+(1-\delta) b]\left(1-\frac{\mu-\beta}{\beta} \xi_{z z}^{G}\right)} \\
& +\frac{\frac{\varepsilon_{r k}}{\varepsilon_{c k}}[1+(2-\delta) b][\beta+b[1+(1-\delta) \beta]]\left(2-\frac{\mu-\beta}{\beta} \xi_{z z}^{G}\right)}{\xi_{c c}\left(\beta \theta^{2}+\frac{\mu-\beta}{v} \frac{\varepsilon_{r k}}{\varepsilon_{c k}} b[1+(1-\delta) b]-\frac{\mu-\beta}{\beta} \beta \theta^{2} \xi_{z z}^{G}\right)+\frac{\varepsilon_{r k}}{\varepsilon_{c k}} b[1+(1-\delta) b]\left(1-\frac{\mu-\beta}{\beta} \xi_{z z}^{G}\right)}
\end{aligned}
$$

Local indeterminacy occurs if $\mathcal{P}(1) / \mathcal{P}(0)<0$ and $\mathcal{P}(-1) / \mathcal{P}(0)<0$. Assume then that $1+(1-\delta) b=0$, i.e. $b=-1 /(1-\delta)$. After simplifiying we get

$$
\begin{aligned}
\frac{\mathcal{P}(1)}{\mathcal{P}(0)} & =\frac{\mu-\beta}{\beta(1-\delta)^{2}} \frac{\frac{\varepsilon_{r k}}{\varepsilon_{c k}} \xi_{z z}^{G}}{\beta \theta^{2} \xi_{c c}\left(1-\frac{\mu-\beta}{\beta} \xi_{z z}^{G}\right)} \\
\frac{\mathcal{P}(-1)}{\mathcal{P}(0)} & =\frac{2 \xi_{c c}\left[\frac{\mu-\beta}{v(1-\delta)^{2}} \frac{\varepsilon_{r k}}{\varepsilon_{c k}}+\beta(1+\beta) \theta^{2}\left(2-\frac{\mu-\beta}{\beta} \xi_{z z}^{G}\right)\right]+\frac{\varepsilon_{r k}}{\varepsilon_{c k}(1-\delta)^{2}}\left(2-\frac{\mu-\beta}{\beta} \xi_{z z}^{G}\right)}{\beta \theta^{2} \xi_{c c}\left(1-\frac{\mu-\beta}{\beta} \xi_{z z}^{G}\right)}
\end{aligned}
$$

Consider first $\mathcal{P}(1) / \mathcal{P}(0)$. The numerator is positive while the denominator is negative, so that $\mathcal{P}(1) / \mathcal{P}(0)<0$. Consider then $\mathcal{P}(-1) / \mathcal{P}(0)$. As the denominator is negative, $\mathcal{P}(-1) / \mathcal{P}(0)<0$ if the numerator is positive. A necessary condition for this is given by the following condition

$$
\frac{\mu-\beta}{v(1-\delta)^{2}} \frac{\varepsilon_{r k}}{\varepsilon_{c k}}+\beta(1+\beta) \theta^{2}\left(2-\frac{\mu-\beta}{\beta} \xi_{z z}^{G}\right)<0
$$

Assume that

$$
\lim _{\delta \rightarrow 1} \frac{\varepsilon_{r k}}{\varepsilon_{c k}(1-\delta)^{2}}=-\infty
$$


Then there exists $\underline{\delta} \in(0,1)$ such that when $\delta \in(\underline{\delta}, 1)$, this inequality is satisfied. We conclude that when $\delta \in(\underline{\delta}, 1)$, the numerator is positive if

$$
\xi_{c c}<-\frac{\frac{\varepsilon_{r k}}{\varepsilon_{c k}(1-\delta)^{2}}\left(2-\frac{\mu-\beta}{\beta} \xi_{z z}^{G}\right)}{2\left[\frac{\mu-\beta}{v(1-\delta)^{2}} \frac{\varepsilon}{r k}_{c k}+\beta(1+\beta) \theta^{2}\left(2-\frac{\mu-\beta}{\beta} \xi_{z z}^{G}\right)\right]} \equiv \tilde{\xi}_{c c}
$$

The result follows.

\subsection{Proof of Theorem 5}

When the utility function assumes the KPR formulation, we have with the CIA timing

$$
\xi_{c c}=-\phi, \xi_{z c}=1+\xi_{c c}, \xi_{c z}=\left(1+\xi_{c c}\right) \frac{\mu-\beta}{\beta v}, \xi_{z z}=\xi_{c z}+\eta
$$

with $\eta=\eta\left(m^{*}\right)<0$. It follows from (48) that

$$
\begin{aligned}
\frac{\mathcal{P}(1)}{\mathcal{P}(0)} & =\frac{\mu-\beta}{\mu} \frac{\frac{\varepsilon_{r k}}{\varepsilon_{c k}}(1-\delta b)\left(\beta-\theta^{-1} b\right) \eta}{\xi_{c c}\left(\beta \theta^{2}+\frac{\mu-\beta}{\beta v} \frac{\varepsilon_{r k}}{\varepsilon_{c k}} b[1+(1-\delta) b]\right)+\left(1+\frac{\mu-\beta}{\beta v}\right) \frac{\varepsilon_{r k}}{\varepsilon_{c k}} b[1+(1-\delta) b]} \\
\frac{\mathcal{P}(-1)}{\mathcal{P}(0)} & =\frac{2 \xi_{c c}\left\{\frac{\mu-\beta}{\beta v} \frac{\varepsilon_{r k}}{\varepsilon_{c k}}[1+(2-\delta) b][\beta+b[1+(1-\delta) \beta]]+\beta(1+\beta) \theta^{2}\left[2+\frac{\mu-\beta}{\mu}\left(\eta-\frac{\mu-\beta}{\beta v}\right)\right]\right\}}{\xi_{c c}\left(\beta \theta^{2}+\frac{\mu-\beta}{\beta v} \frac{\varepsilon_{r k}}{\varepsilon_{c k}} b[1+(1-\delta) b]\right)+\left(1+\frac{\mu-\beta}{\beta v}\right) \frac{\varepsilon_{k}}{\varepsilon_{c k}} b[1+(1-\delta) b]} \\
& +\frac{\frac{\varepsilon_{r k}}{\varepsilon_{c k}}[1+(2-\delta) b][\beta+b[1+(1-\delta) \beta]]\left(2+\frac{\eta(\mu-\beta)}{\mu}+2 \frac{\mu-\beta}{\beta v}\right)-2 \beta(1+\beta) \theta^{2} \frac{(\mu-\beta)^{2}}{\mu \beta v}}{\xi_{c c}\left(\beta \theta^{2}+\frac{\mu-\beta}{\beta v} \frac{\varepsilon_{r k}}{\varepsilon_{c k}} b[1+(1-\delta) b]\right)+\left(1+\frac{\mu-\beta}{\beta v}\right) \frac{\varepsilon_{k} b}{\varepsilon_{c k}} b[1+(1-\delta) b]}
\end{aligned}
$$

Local indeterminacy will be obtained if $\mathcal{P}(1) / \mathcal{P}(0)<0$ and $\mathcal{P}(-1) / \mathcal{P}(0)<0$. Assume that $b \in(-1 /(1-\delta),-1 /(2-\delta))$, and consider first $\mathcal{P}(1) / \mathcal{P}(0)$. As the numerator is positive and $b[1+(1-\delta) b]<0$, the denominator is negative, and thus $\mathcal{P}(1) / \mathcal{P}(0)<0$, if

$$
\xi_{c c}<-\frac{\left(1+\frac{\mu-\beta}{\beta v}\right) \frac{\varepsilon_{r k}}{\varepsilon_{c}} b[1+(1-\delta) b]}{\beta \theta^{2}+\frac{\mu-\beta}{\beta v} \frac{\varepsilon_{r k}}{\varepsilon_{c k}} b[1+(1-\delta) b]} \equiv \xi_{c c}^{1}
$$

Consider then $\mathcal{P}(-1) / \mathcal{P}(0)$. As the denominator is negative, $\mathcal{P}(-1) / \mathcal{P}(0)<$ 0 if the numerator is positive. Consider the term between brackets in the numerator which is multiplied to $\xi_{c c}$. Since $[1+(2-\delta) b][\beta+b[1+(1-\delta) \beta]]>$ 0 , a sufficient condition to get a negative expression is

$$
2+\frac{\mu-\beta}{\mu} \eta \leq 0
$$

If this inequality holds we have $\lim _{\xi_{c c} \rightarrow-\infty} \mathcal{P}(-1) / \mathcal{P}(0)=-\infty$. Therefore, there exists $\xi_{c c}^{2}<0$ such that $\mathcal{P}(-1) / \mathcal{P}(0)<0$ when $\xi_{c c}<\xi_{c c}^{2}$. The result follows by taking $\bar{\xi}_{c c}=\min \left\{\xi_{c c}^{1}, \xi_{c c}^{2}\right\}$. 


\subsection{Proof of Theorem 6}

When the utility function assumes the KPR formulation, we have with the CAM timing

$$
\xi_{c c}=-\phi, \xi_{z c}=1+\xi_{c c}, \xi_{c z}=\left(1+\xi_{c c}\right) \frac{\mu-\beta}{v}, \xi_{z z}=\xi_{c z}+\eta
$$

with $\eta=\eta\left(z^{*}\right)<0$. It follows from (49) that

$$
\begin{aligned}
\frac{\mathcal{P}(1)}{\mathcal{P}(0)} & =\frac{\frac{\mu-\beta}{\beta} \frac{\varepsilon_{r k}}{\varepsilon_{c k}}(1-\delta b)\left(\beta-\theta^{-1} b\right) \eta}{\xi_{c c}\left[\beta \theta^{2}-\frac{(\mu-\beta) \mu}{\beta v} \frac{\varepsilon_{r k}}{\varepsilon_{c k}} b[1+(1-\delta) b]-(\mu-\beta)\left(\eta-\frac{\mu-\beta}{v}\right) \theta^{2}\right]+\frac{\varepsilon_{r k}}{\varepsilon_{c k}} b[1+(1-\delta) b]\left(1-\frac{(\mu-\beta) \mu}{\beta v}\right)+\frac{(\mu-\beta)^{2}}{v} \theta^{2}} \\
\frac{\mathcal{P}(-1)}{\mathcal{P}(0)} & =\frac{2 \xi_{c c}\left\{\frac{\mu-\beta}{v} \frac{\varepsilon_{r k}}{\varepsilon_{c k}}[1+(2-\delta) b][\beta+b[1+(1-\delta) \beta]]+\beta(1+\beta) \theta^{2}\left[2-\frac{\mu-\beta}{\beta}\left(\eta-\frac{\mu-\beta}{v}\right)\right]\right\}}{\xi_{c c}\left[\beta \theta^{2}-\frac{(\mu-\beta) \mu}{\beta v} \frac{\varepsilon_{r k}}{\varepsilon_{c k}} b[1+(1-\delta) b]-(\mu-\beta)\left(\eta-\frac{\mu-\beta}{v}\right) \theta^{2}\right]+\frac{\varepsilon_{r k}}{\varepsilon_{c k}} b[1+(1-\delta) b]\left(1-\frac{(\mu-\beta) \mu}{\beta v}\right)+\frac{(\mu-\beta)^{2}}{v} \theta^{2}} \\
& +\frac{\frac{\varepsilon_{r k}}{\varepsilon_{c k}}[1+(2-\delta) b][\beta+b[1+(1-\delta) \beta]]\left[2+\frac{\mu-\beta}{v}\left(2-\frac{\eta v}{\beta}\right)\right]+2(1+\beta) \theta^{2} \frac{(\mu-\beta)^{2}}{v}}{\xi_{c c}\left[\beta \theta^{2}-\frac{(\mu-\beta) \mu}{\beta v} \frac{\varepsilon_{r k}}{\varepsilon_{c k}} b[1+(1-\delta) b]-(\mu-\beta)\left(\eta-\frac{\mu-\beta}{v}\right) \theta^{2}\right]+\frac{\varepsilon_{r k}}{\varepsilon_{c k}} b[1+(1-\delta) b]\left(1-\frac{(\mu-\beta) \mu}{\beta v}\right)+\frac{(\mu-\beta)^{2}}{v} \theta^{2}}
\end{aligned}
$$

Local indeterminacy will be obtained if $\mathcal{P}(1) / \mathcal{P}(0)<0$ and $\mathcal{P}(-1) / \mathcal{P}(0)<0$. Assume then that $1+(1-\delta) b=0$, i.e. $b=-1 /(1-\delta)$. Straightforward simplifications give

$$
\begin{aligned}
\frac{\mathcal{P}(1)}{\mathcal{P}(0)} & =\frac{\frac{\mu-\beta}{\beta(1-\delta)^{2}} \frac{\varepsilon_{r k}}{\varepsilon_{c k}} \eta}{\xi_{c c} \theta^{2}\left[\beta-(\mu-\beta)\left(\eta-\frac{\mu-\beta}{v}\right)\right]+\frac{(\mu-\beta)^{2}}{v} \theta^{2}} \\
\frac{\mathcal{P}(-1)}{\mathcal{P}(0)} & =\frac{\frac{\varepsilon_{r k}}{\varepsilon_{c k}(1-\delta)^{2}}\left[2+\frac{\mu-\beta}{v}\left(2-\frac{\eta v}{\beta}\right)+2 \xi_{c c} \frac{\mu-\beta}{v}\right]+2 \beta(1+\beta) \theta^{2}\left\{\xi_{c c}\left[2-\frac{\mu-\beta}{\beta}\left(\eta-\frac{\mu-\beta}{v}\right)\right]+\frac{(\mu-\beta)^{2}}{\beta v}\right\}}{\xi_{c c} \theta^{2}\left[\beta-(\mu-\beta)\left(\eta-\frac{\mu-\beta}{v}\right)\right]+\frac{(\mu-\beta)^{2}}{v} \theta^{2}}
\end{aligned}
$$

Consider first $\mathcal{P}(1) / \mathcal{P}(0)$. Since the numerator is positive, $\mathcal{P}(1) / \mathcal{P}(0)<0$ if the denominator is negative, i.e. if

$$
\xi_{c c}<-\frac{\frac{(\mu-\beta)^{2}}{v}}{\beta-(\mu-\beta)\left(\eta-\frac{\mu-\beta}{v}\right)} \equiv \xi_{c c}^{1}
$$

Consider then $\mathcal{P}(-1) / \mathcal{P}(0)$. As the denominator is negative, $\mathcal{P}(-1) / \mathcal{P}(0)<$ 0 if the numerator is positive. Assume that the term between brackets which is pre-multiplied by the ratio $\varepsilon_{r k} / \varepsilon_{c k}$ is negative, namely

$$
\begin{aligned}
& 2+\frac{\mu-\beta}{v}\left(2-\frac{\eta v}{\beta}\right)+2 \xi_{c c} \frac{\mu-\beta}{v}<0 \\
\Leftrightarrow & \xi_{c c}<-\frac{2+\frac{\mu-\beta}{v}\left(2-\frac{\eta v}{\beta}\right)}{2 \frac{\mu-\beta}{v}} \equiv \xi_{c c}^{2}
\end{aligned}
$$

Assume also that

$$
\lim _{\delta \rightarrow 1} \frac{\varepsilon_{r k}}{\varepsilon_{c k}(1-\delta)^{2}}=-\infty
$$

Under these two conditions we get $\lim _{\delta \rightarrow 1} \mathcal{P}(-1) / \mathcal{P}(0)=-\infty$. Therefore, there exists $\underline{\delta} \in(0,1)$ such that $\mathcal{P}(-1) / \mathcal{P}(0)<0$ for any $\delta \in(\underline{\delta}, 1)$. The result follows by taking $\tilde{\xi}_{c c}=\min \left\{\xi_{c c}^{1}, \xi_{c c}^{2}\right\}$. 


\section{References}

[1] Azariadis, C. (1981): "Self Fulfilling Prophecies", Journal of Economic Theory, 25, 380-396.

[2] Barinci, J.P., and A. Chéron (2001): "Real Business Cycles and the Animal Spirits Hypothesis in a CIA Economy," WP 01-13, University of Evry-EPEE.

[3] Becker, R., and E. Tsyganov (2002): "Ramsey Equilibrium in a TwoSector Model with Heterogeneous Households," Journal of Economic Theory, 105, 188-225.

[4] Benhabib, J., and K. Nishimura (1981): "Stability of Equilibrium in Dynamic Models of Capital Theory," International Economic Review, 22, 275-293.

[5] Benhabib, J., and K. Nishimura (1985): "Competitive Equilibrium Cycles," Journal of Economic Theory, 35, 284-306.

[6] Benhabib, J., and K. Nishimura (1998): "Indeterminacy and Sunspots with Constant Returns," Journal of Economic Theory, 81, 58-96.

[7] Bosi, S., and F. Magris (2003): "Indeterminacy and Endogenous Fluctuations with Arbitrarily Small Liquidity Constraint," Research in Economics, 57, 39-51.

[8] Bosi, S., F. Magris and A. Venditti (2005): "Competitive Equilibrium Cycles with Endogenous Labor," Journal of Mathematical Economics, 41, 325-349.

[9] Bosi, S., F. Magris and A. Venditti (2005): "Multiple Equilibria in a Cash-in-Advance Two-Sector Economy," International Journal of Economic Theory, 1, 131-149.

[10] Brock, W. (1974): "Money and Growth: The Case of Long Run Perfect Foresight," International Economic Review, 15, 750-777.

[11] Carlstrom, C., and T. Fuerst (2003): "Money Growth Rules and Price Level Determinacy," Review of Economic Dynamics, 6, 263-275.

[12] Cass, D., and K. Shell (1983): "Do Sunspots Matter ?", Journal of Political Economy, 91, 193-227. 
[13] Clower, R. (1967): "A Reconsideration of the Microfoundations of Monetary Theory," Western Economic Journal, 6, 1-9.

[14] Cooley, T., and G. Hansen (1989): "The Inflation Tax in a Real Business Cycle Model," American Economic Review, 79, 492-511.

[15] Farmer, R. (1997): "Money in a Real Business Cycle Model," Journal of Money, Credit and Banking, 29, 568-611.

[16] Feenstra, R. (1986): "Functional Equivalence Between Liquidity Costs and the Utility of Money," Journal of Monetary Economics, 17, 271291.

[17] Grandmont, J.M., and Y. Younès (1972): "On the Role of Money and the Existence of a Monetary Equilibrium," Review of Economic Studies, 39, 355-372.

[18] Greenwood, J., Z. Hercovitz, and G. Huffman (1988): "Investment, Capacity Utilization and the Real Business Cycle," American Economic Review, 78, 402-417.

[19] Hintermaier, T. (2001): "Lower Bounds on Externalities in Sunspot Models," Working Paper EUI.

[20] Hintermaier, T. (2003): "On the Minimum Degree of Returns to Scale in Sunspot Models of Business Cycles," Journal of Economic Theory, 110, 400-409.

[21] King, R., C. Plosser and S. Rebelo (1988): "Production, Growth and Business Cycles: II. New Directions," Journal of Monetary Economics, 21, 309-341.

[22] Lucas, R., and N. Stokey (1987): "Money and Interest in a Cash-inAdvance Economy," Econometrica, 55, 491-513.

[23] Matsuyama, K. (1990): "Sunspot Equilibria (Rational Bubbles) in a Model of Money-in-the-Utility-Function," Journal of Monetary Economics, 25, 137-144.

[24] Michener, R., and B. Ravikumar (1998): "Chaotic Dynamics in a CashIn-Advance Economy," Journal of Economic Dynamics and Control, 22, 1117-1137.

[25] Pintus, P. (2007): "Local Determinacy with Non-Separable Utility," Journal of Economic Dynamics and Control, 31, 669-682. 
[26] Shell, K. (1977): "Monnaie et Allocation Intertemporelle", mimeo, Séminaire d'Econométrie Roy-Malinvaud, Centre National de la Recherche Scientifique, Paris.

[27] Sidrauski M. (1967): "Rational Choice and Patterns of Growth in a Monetary Economy," American Economic Review, 57, 534-544.

[28] Stockman, A. (1981): "Anticipated Inflation and the Capital Stock in a Cash-in-Advance Economy," Journal of Monetary Economics, 8, 387393.

[29] Takahashi, H., K. Mashiyama and T. Sakagami 2003: "Measuring Capital Intensity in the Postwar Japanese Economy: a Two-Sector Approach", Mimeo, Meiji-Gakuin University, Tokyo.

[30] Woodford, M. (1986): "Stationary Sunspot Equilibria. The Case of Small Fluctuations Around a Deterministic Steady State," Mimeo, University of Chicago and New York University.

[31] Woodford, M. (1994): "Monetary Policy and Price Level Determinacy in a Cash-in-Advance Economy," Economic Theory, 4, 345-380. 